NASA Technical Memorandum 110222

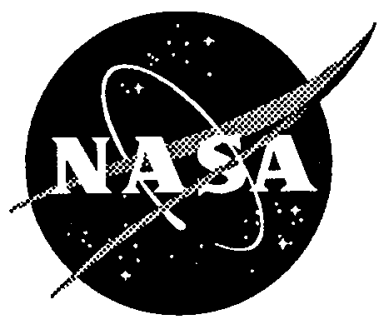

\title{
Computational Considerations for the Simulation of Shock-Induced Sound
}

Jay Casper

Old Dominion University, Norfolk, Virginia

Mark H. Carpenter

Langley Research Center, Hampton, Virginia

December 1995

National Aeronautics and

Space Administration

Langley Research Center

Hampton, Virginia 23681-0001 


\title{
COMPUTATIONAL CONSIDERATIONS FOR THE SIMULATION OF SHOCK-INDUCED SOUND
}

\author{
Jay Casper* \\ Old Dominion University \\ Norfolk, Virginia
}

\author{
Mark H. Carpenter ${ }^{\dagger}$ \\ NASA Langley Research Center \\ Hampton, Virginia
}

\begin{abstract}
The numerical study of aeroacoustic problems places stringent demands on the choice of a computational algorithm, because it requires the ability to propagate disturbances of small amplitude and short wavelength. The demands are particularly high when shock waves are involved, because the chosen algorithm must also resolve discontinuities in the solution. The extent to which a high-order-accurate shock-capturing method can be relied upon for aeroacoustics applications that involve the interaction of shocks with other waves has not been previously quantified. Such a study is initiated in this work. A fourth-order-accurate essentially nonoscillatory (ENO) method is used to investigate the solutions of inviscid, compressible flows with shocks in a quasi-one-dimensional nozzle flow. The design order of accuracy is achieved in the smooth regions of a steady-state test case. However, in an unsteady test case, only first-order results are obtained downstream of a sound-shock interaction. The difficulty in obtaining a globally high-order-accurate solution in such a case with a shock-capturing method is demonstrated through the study of a simplified, linear model problem. Some of the difficult issues and ramifications for aeroacoustic simulations of flows with shocks that are raised by these results are discussed.
\end{abstract}

\footnotetext{
"Research Assistant Professor, Department of Aerospace Engineering, Old Dominion University, Norfolk, VA 23529; supported by U.S. Government grant NAG1-1653.

${ }^{\dagger}$ Research Engineer, Fluid Mechanics and Acoustics Division, NASA Langley Research Center, Hampton, VA 23681.
} 


\section{INTRODUCTION}

This work is motivated by the desire to develop numerical methods that will be useful in the study of aeroacoustic phenomena that occur in flows with shocks. For example, shocks in jet flows, on wings, and in supersonic combustion inlets contribute significantly to sound generation. Problems such as these represent some of the more challenging aspects of ongoing research in the developing area of computational aeroacoustics (CAA).

One of the purposes of this work is to open a discussion on the relative merits of the numerical methods which can simulate sound sources that are generated in flows with shocks. For a computational algorithm, obtaining acoustic information from a numerical solution that involves shock waves is a demanding proposition. In general, high-order accuracy is required for the propagation of high-frequency low-amplitude waves. In addition, the shock must be adequately captured. A significant body of work has been devoted to the development of numerical schemes that possess both properties.

The contribution of a shock wave to the generation of sound is attributed to the motion of the shock wave and its interaction with other disturbances in the flow. These disturbances can be large, such as changes in the mean flow, or a small, such as an acoustic or vortical disturbances. The extent to which such an interaction must be accurately predicted in order to propagate reliable information to an observer will be investigated.

In the following section, some of the currently available methods that vary in their approach to high-order accuracy and shock capturing are briefly surveyed. Next, a fourth-order-accurate essentially nonoscillatory (ENO) method is applied to the steady solution of a nozzle flow with a shock, and fourth-order-accurate results are obtained. However, in the application of the same numerical scheme to a time-dependent problem, that of a sound-shock interaction, accuracy suffers. The disappointing results in regard to the accuracy of this solution are explained through the study of a simpler linear model problem. The numerical scheme is modified with a subcell resolution technique in order to obtain a globally high-order-accurate solution for time-dependent simulations.

The original sound-shock interaction problem is again solved with the modified scheme, and 
a fourth-order-accurate solution is achieved. Some of the difficult issues and ramifications for current methods that are raised by these results are discussed in the final section.

\section{HIGH-ORDER ACCURATE SHOCK-CAPTURING METHODS}

The high-order-accurate spatial operator that is desired in a shock-capturing method for CAA problems requires that information be taken from a large number of discrete locations within the solution. Such an operator will cause large oscillations in a discontinuous solution unless special precautions are taken. Many methods are available in the literature that attempt to balance the properties of high-order accuracy and shock capturing. However, they can be classified into two basic categories i.e., linear and nonlinear.

Within the linear class of numerical shock-capturing schemes, the interpolation set for the approximation of the solution or its derivatives is fixed as a function of grid location. Linear methods admit oscillations in regions in which physical gradients are inadequately resolved. Centraldifferencing operators and spectral methods are particularly prone to these numerical oscillations. For nonlinear problems, limiters or filters are usually required to keep oscillations from growing without bound. The general features of these ideas are demonstrated in the work of Don, ${ }^{1}$ in which pseudospectral methods are used for compressible flow problems with shocks by locally applying a simple filter to keep the solution bounded during the computation. The final solution is postprocessed in order to remove the oscillatory information that developed in time. Nothing in this approach mandates the use of pseudospectral techniques; other higher-order schemes (e.g., centraldifference or compact schemes ${ }^{2}$ ) could also be used. For example, Harten and Chakravarthy ${ }^{3}$ suggest a polynomial interpolation procedure in which the coefficients of the polynomial are limited by a switch that makes a coefficient very small if the corresponding solution derivative is discontinuous.

In the nonlinear class of schemes, the strategy with respect to discontinuities is to employ some sort of adaptive interpolation. The goal is to achieve formal high-order accuracy in smooth regions and high shock resolution without oscillations. The class of ENO schemes ${ }^{4,5}$ has been designed to 
have such properties. As originally presented, the local polynomial approximation operator adapts its interpolation set to the smoothest available part of the solution. This adaptive interpolation strategy has also been employed in the development of psuedospectral hybrid schemes. ${ }^{6-8}$

Although discontinuous solutions generated by a linear strategy are usually not as pictorially pleasing as solutions in which shock profiles are monotone, these schemes are more computationally efficient than nonlinear schemes. The efficiency of a numerical algorithm is extremely important for aeroacoustic simulations because such problems are time dependent and require a fine computational mesh for the resolution of high-frequency disturbances. Because nonlinear methods are designed to avoid the production of spurious oscillations, the stability of a calculation of a flow with shocks is more readily obtained. However, their adaptive interpolation operator significantly hampers their efficiency relative to linear schemes.

In the literature to date, the design accuracy of a numerical method, whether linear or nonlinear, has been demonstrated with solutions to smooth problems. Problems with discontinuous solutions are most commonly used only to illustrate the ability of the scheme to obtain a stable solution to such problems. In the following two sections, the ability of a fourth-order accurate ENO method to achieve high-order accuracy in the smooth regions of a flow with a shock is investigated. The method is applied to obtain the solutions of a steady problem and a time-dependent sound-shock interaction. The accuracies of these solutions are then analyzed through the study of a simpler linear model problem.

\section{STEADY SHOCK IN A NOZZLE}

A steady-state flow with a shock in a quasi-one-dimensional converging-diverging nozzle is numerically investigated. The governing equations are the quasi-one-dimensional Euler equations:

$$
\frac{\partial}{\partial t}(A U)+\frac{\partial}{\partial x}(A F)=G
$$

where

$$
U=\left[\begin{array}{c}
\rho \\
\rho u \\
\rho E
\end{array}\right], F=\left[\begin{array}{c}
\rho u \\
\rho u^{2}+P \\
(\rho E+P) u
\end{array}\right], G=\left[\begin{array}{c}
0 \\
P \frac{d A}{d x} \\
0
\end{array}\right]
$$


The variables $\rho, u, P, E$, and $A$ are the density, velocity, pressure, total specific energy, and nozzle area, respectively. The equation of state is

$$
P=(\gamma-1) \rho\left(E-\frac{1}{2} u^{2}\right)
$$

where $\gamma$ is the ratio of specific heats, which is assumed to have a constant value of 1.4.

The spatial domain of the nozzle is $0 \leq x \leq 1$, and the flow is oriented from left to right. The nozzle shape is determined exactly through the requirement of a linear distribution of Mach number from $M=0.8$ at the inlet to $M=1.8$ at the exit, assuming the flow is isentropic and fully expanded. The resulting area distribution $A(x)$ is illustrated in Fig. 1a. The flow variables are normalized with respect to stagnation conditions at $-\infty$ and the area with respect to the value at the throat $x=0.2$.

Given the prescribed area distribution, the Mach 0.8 inflow state is retained at $x=0$, and the outflow condition at $x=1$ is determined such that a shock forms at $x_{s}=0.5$, which corresponds to a preshock Mach number of $M=1.3$. A steady-state solution is obtained by implementing a fourth-order finite-volume ENO scheme until residuals are driven to machine zero. Spatial accuracy is achieved by solving the equations in control-volume form as presented in Ref. 4. The equations are integrated in time via a third-order-accurate Runge-Kutta scheme. ${ }^{5}$ This numerical method will be referred to as "ENO-4-3." As has been established in previous research, ${ }^{9-12}$ the adaptive stencils employed in the spatial operator are biased in smooth regions toward those that are linearly stable. Fig. $1 \mathrm{~b}$ depicts the solution, with respect to Mach number, on a uniform mesh of 128 cells.

One of the simpler methods of determining the error of this solution relies on the fact that the value of the entropy-like quantity $P / \rho^{\gamma}$ is piecewise constant:

$$
S \equiv \frac{P}{\rho^{\gamma}}=\left\{\begin{array}{l}
S_{-\infty}, \quad x<x_{s} \\
S_{\infty}, \quad x>x_{s}
\end{array}\right.
$$

The subscripts of $S$ denote the pre-shock and post-shock stagnation values, respectively. This quantity is plotted in Fig. 1c. The pointwise entropy error for this solution on four successively refined meshes is illustrated in Fig. 2. Clearly, the accuracy is fourth order on either side of the shock, as demonstrated by the error data in Table I. The variable $N_{c}$ is the number of cells. The 
errors $\|e\|$ are computed in the $L_{1}$ and $L_{\infty}$ norms. The number $r_{c}$ is the computational order of accuracy and is determined by the slope of these tabulated values on a log-log plot:

$$
r_{c}=\frac{\ln \left(e_{h_{k}} / e_{h_{k+1}}\right)}{\ln \left(h_{k} / h_{k+1}\right)}
$$

where $e_{h_{k}}$ is the error measured on a mesh of uniform spacing $h_{k}$ with $h_{k}>h_{k+1}$ for $k=1,2,3$.

Although these results are encouraging, the time independence of the solution makes this a convenient example for the demonstration of high-order accuracy in the presence of a shock. As will be shown in the study of an unsteady problem in the following section, a moving shock presents a greater challenge in regard to high-order-accurate shock capturing.

\section{SOUND-SHOCK INTERACTION}

The interaction of a sound wave with a shock in a one-dimensional flow is numerically investigated. The effects of shocks on sound waves, and vice versa, are important to the acoustics and performance of aircraft design. Therefore, the ability to obtain an accurate solution to such a model initial-boundary-value problem (IBVP) is important in the development of shock-capturing methods for CAA research. Similar one-dimensional problems have been the subject of other studies. ${ }^{13,14}$

The governing equations are the one-dimensional Euler equations:

$$
\frac{\partial}{\partial t} U+\frac{\partial}{\partial x} F(U)=0
$$

where the components of $U$ and $F(U)$ are identical to those given in Eq. 1b. The equation of state is also the same as in the previous example.

The spatial domain is $0 \leq x \leq 1$. The piecewise constant initial conditions, $U_{L}$ and $U_{R}$, are those of a steady shock located at $x_{s}=0.5$. The flow is from left to right, and the state $U_{L}$ is a Mach 2 flow upstream of the shock. The flow variables are normalized with respect to this upstream flow. At $t=0$, an acoustic disturbance is introduced at $x=0$ : 


$$
\begin{aligned}
P(0, t) & =P_{L}(1+\epsilon \sin \omega t) \\
\rho(0, t) & =\rho_{L}\left[\frac{P(0, t)}{P_{L}}\right]^{1 / \gamma} \\
u(0, t) & =u_{L}+\frac{2}{\gamma-1}\left[c(0, t)-c_{L}\right]
\end{aligned}
$$

where $\omega$ is the circular frequency, $\epsilon$ is the amplitude, and $c=\sqrt{\gamma P / \rho}$ is the local sound speed.

The numerical solution of this problem is obtained through the implementation of the ENO-4-3 algorithm. This algorithm will be fourth-order accurate even for a time-dependent problem when the time step is suitably restricted. The exact solution is obtained by a two-domain Chebyshev spectral technique. ${ }^{15}$ Shock fitting is used to divide the domain into two computational regions. A Chebyshev collocation method is used in each region for the spatial discretization. A fourth-order Runge-Kutta scheme is used to discretize time. Sufficient spatial and temporal resolution are used to guarantee machine precision of the solution.

Fig. 3 depicts the pressure perturbation $\delta P(t)=P(x, t)-P(x, 0)$ at $t=30 T_{\lambda}$, where $T_{\lambda}=2 \pi / \omega$ is one period of the incoming acoustic wave. The acoustic wave amplitude is $\epsilon=0.001$, and $\omega=2 \pi k\left(u_{L}+c_{L}\right)$ is determined by requiring a wave number $k=6$ with respect to unit length and a mean wave speed $u_{L}+c_{L}$. The calculation, represented by circles, was performed on a uniform mesh of 256 cells with a Courant number of 0.5 . The exact solution is represented by a continuous line. In this pictorial measure, the numerical algorithm performs well with respect to its prediction of the amplified sound wave at higher frequency downstream of the shock. The missing circle values near the shock are off the plot and are due to the use of the stencil-biasing parameters near a moving discontinuity.

Even more instructive, however, is the pointwise error made by this calculation with respect to the mesh width. Fig. 4 illustrates this error on four successively refined meshes. The solution is clearly fourth-order accurate upstream of the shock, but only first-order downstream of the shock, as shown by the $L_{\infty}$ error data in Table II. The errors are computed on two spatial subdomains: $0 \leq x \leq 0.45$ and $0.55 \leq x \leq 1$. In this manner, the first-order error that is generated in the 
vicinity of the shock is avoided. This disappointing result is more easily explained through the study of a simpler model problem, which is examined in the following section.

\section{A LINEAR MODEL PROBLEM}

The lower-order accurate results of the previous section can be analyzed through the study of a linear scalar model problem. The solution of the following IBVP is instructive because it isolates the important phenomenon of propagation of information through a discontinuity. This trait will be common to almost any aeroacoustic problem that involves shock waves. Consider the scalar equation:

$$
\frac{\partial u}{\partial t}+a(x) \frac{\partial u}{\partial x}=0
$$

where the piecewise constant wave speed $a(x)$ is

$$
a(x)= \begin{cases}2, & x \leq x_{s} \\ 1, & x>x_{s}\end{cases}
$$

The initial conditions are chosen as

$$
u(x, 0)= \begin{cases}\frac{1}{2}, & x \leq x_{s} \\ 1, & x>x_{s}\end{cases}
$$

The spatial domain is $0 \leq x \leq 1$, and the discontinuity location is $x_{s}=0.5$. The inflow boundary condition is

$$
u(0, t)=\frac{1}{2}(1+\epsilon \sin \omega t)
$$

with $\epsilon=0.001$ and $\omega$ determined by requiring a wave number $k=2$ with respect to unit length and the upstream wave speed $a=2$.

For the purpose of the subsequent discussion, it is necessary to briefly describe the numerical scheme. The semi-discrete, finite-volume formulation is obtained by integrating Eq. $3 \mathrm{a}$ on an interval $\left[x_{i-1 / 2}, x_{i+1 / 2}\right]$ with center $x_{i}$ and "volume" $\Delta x_{i}$ :

$$
\frac{\partial}{\partial t} \bar{u}_{i}(t)=\frac{-1}{\Delta x_{i}}\left[\hat{f}_{i+1 / 2}(t)-\hat{f}_{i-1 / 2}(t)\right]
$$


where

$$
\bar{u}_{i}(t)=\frac{1}{\Delta x_{i}} \int_{x_{i-1 / 2}}^{x_{i+1 / 2}} u(x, t) d x
$$

is the cell average of $u$ on the $i$ th interval at time $t$, and the flux $\hat{f}_{i+1 / 2}(t)$ is

$$
\hat{f}_{i+1 / 2}(t)=a\left(x_{i+1 / 2}\right) u\left(x_{i+1 / 2}, t\right)
$$

Temporal integration is achieved by a Runge-Kutta method. ${ }^{5}$ A numerical approximation to the flux in Eq. $4 \mathrm{c}$ is determined in two steps. First, given the cell averages, the solution is approximated pointwise within each cell.

$$
\mathcal{P}_{i}(x) \approx u(x, t), \quad x_{i-1 / 2} \leq x \leq x_{i+1 / 2}
$$

In this particular application, $\mathcal{P}_{i}(x)$ is a cubic polynomial. The process by which $\mathcal{P}_{i}(x)$ is obtained will be referred to as "reconstruction." This reconstruction operator contains the adaptive stencil algorithm, which avoids interpolation across steep gradients. (See Ref. 4 for details.) Then, at a cell interface $x_{i+1 / 2}$, two solution values exist, as shown in Fig. 5. Correspondingly, two flux values also exist. The second step, then, is simply to choose the upwind value which, for the present problem, yields the following numerical flux approximations:

$$
\begin{aligned}
& \hat{f}_{i-1 / 2}(t) \approx a\left(x_{i-1 / 2}\right) \mathcal{P}_{i-1}\left(x_{i-1 / 2}\right) \\
& \hat{f}_{i+1 / 2}(t) \approx a\left(x_{i+1 / 2}\right) \mathcal{P}_{i}\left(x_{i+1 / 2}\right)
\end{aligned}
$$

Fig. 6 depicts the perturbation $\delta u(t)=u(x, t)-u(x, 0)$ at $t=10 T_{\lambda}$, with the ENO-43 algorithm. Note the similarity of the features of this solution to those of the sound-shock interaction in regard to the changes in amplitude and frequency across the discontinuity. The calculation, represented by circles, was performed on a uniform mesh of 64 cells with a Courant number of 0.5 . The exact solution is

$$
u(x, t)= \begin{cases}\frac{1}{2}[1+\epsilon \sin \omega(t-x / 2)], & 0 \leq x \leq x_{s} \\ 1+\epsilon \sin \omega\left(t-x+x_{s}\right), & x_{s}<x \leq 1\end{cases}
$$

for $t \geq 3 / 4$, after the perturbation first reaches $x=1$.

Because of the linearity of this problem, the discontinuity location $x_{s}$ remains fixed for all time, unlike the sound-shock interaction problem. Therefore, $x_{s}$ can be conveniently placed with 
respect to a given mesh. For instance, if the computational mesh is composed of an even number of uniform cells, then $x_{s}=0.5$ will lie on a grid point (i.e., a cell face). Fig. 7a depicts the pointwise computational error for this problem on four successively refined meshes for which $x_{s}$ lies on a grid point. The numerical solution is fourth-order accurate throughout the domain, as shown by the error data in Table III. However, for an odd number of uniform cells, $x_{s}$ is interior to a cell, and the solution is first-order accurate downstream of the discontinuity, as shown in Fig. 7b and Table IV.

The strikingly different results in Figs. $7 \mathrm{a}$ and $7 \mathrm{~b}$ yield insight into the disappointing results for the sound-shock interaction problem of the previous section. Even if the initial steady shock is located on a cell face, it moves into the interior of a cell upon interaction with the upstream acoustic wave. Therefore, the following explanation for the lower-order accurate results focuses on the location of the discontinuity with respect to the boundary or interior of a cell.

The difference in the results in Figs. $7 \mathrm{a}$ and $7 \mathrm{~b}$ can be explained in terms of the interpolation operator and its direct influence upon the numerical flux. First, consider the case in which the discontinuity is on a grid point, for example, the left-hand endpoint of the $i$ th cell, as shown in Fig. 8a. Because of the adaptive interpolation, the polynomial approximation within the adjoining cells is of pointwise, high-order accuracy. The large jump at the left-hand interface of the $i$ th cell is immaterial because the flux is determined by the value $\mathcal{P}_{i-1}\left(x_{i-1 / 2}\right)$ and the upstream wave speed $a=2$, as given by Eq. 4e. Now, consider the case in which $x_{s}$ is in the interior of the $i$ th cell, as in Fig. 8b. Unlike Fig. 8a, the polynomial approximation in the $i$ th cell now contains a pointwise error that is $O(1)$. This still does not affect the flux into the $i$ th cell, because the inbound flux is determined only by information in the $(i-1)$ th cell. However, the outbound flux is influenced by the value $\mathcal{P}_{i}\left(x_{i+1 / 2}\right)$, which is ultimately responsible for the large errors downstream of the discontinuity.

The loss in accuracy in numerical solutions of linear problems with discontinuous initial data has been the subject of previous research by other authors. ${ }^{16-19}$ All of these previous studies involved solutions of coupled linear systems. It is, therefore, instructive to note that the solution of 
IBVP 3 is analogous to that of a coupled system in the following way. Let $u_{1}$ denote the solution of Eqs. 3a-d on $0 \leq x \leq x_{s}$, and $u_{2}$ denote the solution on $x_{s}<x \leq 1$. Now consider, for $\delta>0$, the solution $u_{2}$ on $x_{s}<x<x_{s}+\delta$. The downstream solution $u_{2}$ is coupled to $u_{1}$ by the requirement of flux conservation:

$$
\lim _{\delta \rightarrow 0} u_{2}\left(x_{s}+\delta, t\right)=a\left(x_{s}\right) u_{1}\left(x_{s}, t\right)
$$

As in the present study, the work of Majda and Osher ${ }^{16}$ was concerned with the inherent degradation in accuracy in a region in which information must be numerically propagated across a discontinuity. Majda and Osher suggested that this difficulty could be circumvented by smoothing the initial data. (See Ref. 16 for details.) However, because the goal of the present work is the application of numerical methods to solutions of nonlinear problems, the approach suggested by Donat and Osher ${ }^{19}$ is more appropriate to use here. These authors propose to maintain accuracy across discontinuities by using Harten's ideas on subcell resolution. ${ }^{20}$

In an attempt to achieve a globally high-order-accurate solution, a subcell resolution technique is considered. In particular, a modification of the procedure presented in Ref. 20 is detailed. The goal is to obtain a better pointwise approximation of the outbound flux in the $i$ th cell in Fig. $8 \mathrm{~b}$. If the $i$ th cell is decomposed into two subcells whose adjacent face is $x_{s}$, then the polynomial approximation $\mathcal{P}_{i}(x)$ would be replaced with the piecewise polynomial approximation

$$
\tilde{\mathcal{P}}_{i}(x)= \begin{cases}\mathcal{P}_{L}(x), & x_{i-1 / 2} \leq x<x_{s} \\ \mathcal{P}_{R}(x), & x_{s}<x \leq x_{i+1 / 2}\end{cases}
$$

where $\mathcal{P}_{L}(x)$ and $\mathcal{P}_{R}(x)$ approximate $u(x, t)$ in the $i$ th cell to high-order pointwise accuracy on either side of the discontinuity. Because the inbound flux is accurately approximated with the polynomial in the $(i-1)$ th cell, $\mathcal{P}_{L}(x)$ can be determined by simple extrapolation of $\mathcal{P}_{i-1}(x)$, as shown in Fig. 9. With this reasoning, for the present piecewise cubic polynomial reconstruction, clearly

$$
\bar{u}_{i}(t)=\frac{1}{\Delta x_{i}}\left[\int_{x_{i-1 / 2}}^{x_{s}} \mathcal{P}_{i-1}(x) d x+\int_{x_{s}}^{x_{i+1 / 2}} \mathcal{P}_{R}(x) d x\right]+O\left(\Delta x^{4}\right)
$$

Finally, $\mathcal{P}_{R}(x)$ must be accurately determined, which enables an accurate outbound flux calculation given by $\mathcal{P}_{R}\left(x_{i+1 / 2}\right)$. Let $\bar{u}_{R}$ denote the subcell average of $\mathcal{P}_{R}(x)$ on $\left[x_{s}, x_{i+1 / 2}\right]$. Then, 
clearly, from Eq. 7,

$$
\bar{u}_{R}=\frac{1}{x_{i+1 / 2}-x_{s}}\left[\Delta x_{i} \bar{u}_{i}(t)-\int_{x_{i-1 / 2}}^{x_{s}} \mathcal{P}_{i-1}(x) d x\right]+O\left(\Delta x^{4}\right)
$$

The desired cubic polynomial $\mathcal{P}_{R}(x)$ is then determined by applying the reconstruction operator to the set $\left\{\bar{u}_{R}, \bar{u}_{i+1}(t), \bar{u}_{i+2}(t), \bar{u}_{i+3}(t)\right\}$, which contains only smooth solution information.

Note that, for the solution of the present problem, the outbound flux cannot be determined by the leftward extrapolation of $\mathcal{P}_{i+1}(x)$, as in Ref. 20 . This method would be appropriate only if the initial conditions contained the propagating wave profile. In the current problem, at $t=0$, the solution is constant downstream of $x_{s}$. Therefore, the extrapolation of $\mathcal{P}_{i+1}(x)$ into the $i$ th cell would never allow the wave to propagate into the $(i+1)$ th cell.

The subcell resolution method described above was incorporated into the ENO algorithm, and the linear model problem was again solved with the discontinuity located in the interior of a cell. This modified scheme will be referred to as "ENO-4-3-SR." The globally high-order-accurate results are shown by the pointwise error plot in Fig. 10 and Table V.

\section{SOUND-SHOCK INTERACTION: REVISITED}

The sound-shock interaction problem is again solved; this time the subcell resolution methodology from the previous section is incorporated. However, the subcell technique applied in the linear problem above does not immediately carry over to this nonlinear case. The method in Eqs. 6-8 is dependent upon the discontinuity location $x_{s}$. For the nonlinear case, at a given time $t$, the shock location is not known a priori and, therefore, must be determined before the subcell technique in Eqs. 6-8 can be applied. This step can be accomplished by solving $H\left(x_{s}\right)=0$ for $x_{s}$ in the interval $\left(x_{i-1 / 2}, x_{i+1 / 2}\right)$ where the function $H\left(x_{s}\right)$ is given by

$$
H\left(x_{s}\right)=\frac{1}{\Delta x_{i}}\left[\int_{x_{i-1 / 2}}^{x_{s}} \mathcal{P}_{i-1}(x) d x+\int_{x_{s}}^{x_{i+1 / 2}} \mathcal{P}_{i+1}(x) d x\right]-\bar{u}_{i}(t)
$$

Clearly, this function is derived from the relationship in Eq. 7.

For the current ENO-4-3-SR algorithm, $H\left(x_{s}\right)$ is a quartic polynomial. For a sufficiently small $\Delta x_{i}$, a unique real root of $H\left(x_{s}\right)$ exists in the interval $\left(x_{i-1 / 2}, x_{i+1 / 2}\right)$. The root of $H\left(x_{s}\right)$ in the 
$i$-th cell is determined numerically by interval halving; the interval midpoint is taken as the initial guess. After the shock location $x_{s}$ is known, the subcell resolution discussed in the previous section is applied to the Euler equations in a component-by-component manner. Figure 11 illustrates the pointwise solution error determined by the modified ENO algorithm on four successively refined grids. The solution converges at the same rate downstream of the shock as it does upstream, as shown by the $L_{\infty}$ errors in Table VI.

\section{DISCUSSION}

The foregoing results raise several issues in regard to the development and application of highorder-accurate shock-capturing methods. Although the results presented here were obtained with a high-order ENO method, they are consistent with those obtained with linear high-order schemes. These issues indicate the need for further investigation into the relative merits of high-order-accurate shock-capturing schemes.

The observation that is, perhaps, the most discouraging is the apparent complexity in achieving high-order accuracy in the presence of moving shocks. There are at least two significant drawbacks to the inclusion of subcell resolution in a numerical algorithm. The first is the added cost. Although the additional expense for the subcell resolution was relatively minor for the sound-shock interaction problem, this modification does not suffice for the more general case. For example, the existence and location of a single shock was assumed to be known a priori. This assumption was valid because of the assuredness that the shock would not move outside the initial cell location, within the required sound-wave amplitude range and mesh-refinement parameterization. However, in the more general case, every cell must be tested for the harboring of a discontinuity before the subcell resolution can be applied. (Such a test is proposed in Ref. 20.) The second and more significant drawback to this approach is that its extension to multiple dimensions is not straight forward. A shock is a curve in two-dimensional space, and a surface in three dimensions. The multiple parameterization required to extend Eq. 9 to two- and three-dimensional problems would be cumbersome, to say the least, and would still not guarantee a unique solution for the shock 
location and shape.

These observations raise considerable concern in regard to the use of high-order-accurate methods in the study of unsteady flows with shocks. If high-order methods only yield first-order results, why use them? Before this question can be fully answered, it must first be determined whether the first-order error from a high-order method is significantly smaller than that of a lower order method. This determination must also be balanced with a concern for computational efficiency. This question is a topic for future research.

The ENO methods applied in the present work are designed to capture shocks narrowly without oscillations. However, this property alone was found insufficient to produce globally high-order results in the solution of an unsteady problem, which suggests that the nonoscillatory properties that guarantee the convergence of a nonlinear scheme do not also guarantee the order of that convergence. If this is the case, why not use a linear method? How important is the control of a solution's total variation from the viewpoint of requiring an answer to a given problem? Is it sufficient to simply keep a given computation stable in order to extract the desired information from a solution? Further research is necessary in regard to the relative merits of linear and nonlinear schemes as they are applied to unsteady flows with shocks. This research should include experiments that compare the solution errors of linear and nonlinear schemes with respect to their shock resolution, design accuracy, and computational efficiency. 


\section{REFERENCES}

1. Don, W.S., "Numerical Study of Pseudospectral Methods in Shock Wave Applications," Journal of Computational Physics, Vol. 110, No. 1, January, 1994, pp. 103-111.

2. Lele, S.K., "Compact Finite Different Schemes with Spectral-Like Resolution," Journal of Computational Physics, Vol. 103, 1992, pp. 16-42.

3. Harten, A. and Chakravarthy, S., "Multi-Dimensional ENO Schemes for General Geometries," NASA Contractor Report 187637, ICASE Report No. 91-76, September, 1991.

4. Harten, A., Engquist, B., Osher, S., and Chakravarthy, S., "Uniformly High Order Accurate Essentially Non-Oscillatory Schemes III," Journal of Computational Physics, Vol. 71, No. 2, 1987, pp. 231-323.

5. Shu, C. and Osher, S., "Efficient Implementation of Essentially Non-Oscillatory Shock-Capturing Schemes," Journal of Computational Physics, Vol. 77, No. 2, 1988, pp. 439-471.

6. Cai, W.C., Gottlieb, D., Shu, C.W., "Essentially Non-Oscillatory Spectral Fourier Methods for Shock Wave Calculations," Math. Comp., Vol. 52, No. 186, 1989, pp. 389-410.

7. Cai, W.C., Shu, C.W., "Uniform High-Order Spectral Methods for One and Two-Dimensional Euler Equations," Journal of Computational Physics, Vol. 104, February, 1993, pp. 427-443.

8. Suresh, A., "An Assessment of Spectral Non-Oscillatory Schemes," Journal of Computational Physics, Vol. 114, 1994, pp. 339-349.

9. Rogerson, A. and Meiberg, E. "A Numerical Study of the Convergence Properties of ENO Schemes," Journal of Scientific Computing, Vol. 5, No. 2, 1990, pp. 151-167.

10. Shu, C., "Numerical Experiments on the Accuracy of ENO and Modified ENO Schemes," Journal of Scientific Computing, Vol. 5, No. 2, 1990, pp. 127-150.

11. Atkins, H., "High-Order ENO Methods for the Unsteady Navier-Stokes Equations," AIAA 91-1557, June, 1991. 
12. Casper, J., Shu, C. W., and Atkins, H., “A Comparison of Two Formulations for High-Order Accurate Essentially Non-Oscillatory Schemes," AIAA Journal, Vol. 32, No. 10, pp. 1970-1977, October, 1994.

13. Meadows, K., Caughey, D., and Casper, J., “Computing Unsteady Shock Waves for Aeroacoustic Applications," AIAA Journal, Volume 32, No. 7, pp. 1360-1366, July, 1994.

14. Casper, J. and Meadows, K.R., "Using High-Order Accurate Essentially Non-Oscillatory Schemes for Aeroacoustic Applications ," AIAA 95-0163, January, 1995.

15. Carpenter, M. H., Atkins, H. L. and Singh, D. J., "Characteristic and Finite-Wave ShockFitting Boundary Conditions for Chebyshev Methods," Transition, Turbulence, and Combustion, Vol. II, M. Y. Hussaini, T. B. Gatski, and T. L. Jackson, eds., Kluwer Academic Publishers, 1994.

16. Majda, A. and Osher, S., "Propagation of Error in Regions of Smoothness for Accurate Difference Approximations to Hyperbolic Equations," Comm. Pure and Applied Math, Vol. 30, 1977, pp. $671-705$.

17. Mock, M. S. and Lax, P. D., “The Computation of Discontinuous Solutions of Linear Hyperbolic Equations," Comm. Pure and Applied Math, Vol. 31, 1978, pp. 423-430.

18. Shu, C. W., "Numerical Solutions of Conservation Laws," Ph. D. Dissertation, UCLA, 1986.

19. Donat, R. and Osher, S., "Propagation of Error in Regions of Smoothness for Nonlinear Approximations to Hyperbolic Equations," Computer Methods in Applied Mechanics and Engineering, Vol. 80, 1990 , pp. 59-64.

20. Harten, A., “ENO Schemes with Subcell Resolution,” Journal of Computational Physics, Vol. 83,1989 , pp. $148-184$. 
Table I. Steady-State Entropy Errors

\begin{tabular}{ccccc}
\hline \hline \multicolumn{5}{c}{ ENO-4-3 } \\
$N_{c}$ & $\|e\|_{1}$ & $r_{c}$ & $\|e\|_{\infty}$ & $r_{c}$ \\
\hline 32 & $2.736 \mathrm{E}-07$ & & $4.154 \mathrm{E}-06$ \\
64 & $1.800 \mathrm{E}-08$ & 3.93 & $4.370 \mathrm{E}-07$ & 3.25 \\
128 & $1.085 \mathrm{E}-09$ & 4.05 & $3.671 \mathrm{E}-08$ & 3.57 \\
256 & $6.372 \mathrm{E}-11$ & 4.09 & $2.697 \mathrm{E}-09$ & 3.77 \\
\hline
\end{tabular}

Table II. $L_{\infty}$ Pressure Errors for IBVP 2

\begin{tabular}{ccccc}
\hline \hline \multicolumn{4}{c}{ ENO-4-3 } \\
$N_{c}$ & $x \leq 0.45$ & $r_{c}$ & $x \geq 0.55$ & $r_{c}$ \\
\hline 64 & $8.358 \mathrm{E}-05$ & & $1.677 \mathrm{E}-03$ \\
128 & $6.540 \mathrm{E}-06$ & 3.68 & $1.392 \mathrm{E}-04$ & 3.59 \\
256 & $4.758 \mathrm{E}-07$ & 3.78 & $3.087 \mathrm{E}-05$ & 2.17 \\
512 & $4.511 \mathrm{E}-08$ & 3.40 & $1.689 \mathrm{E}-05$ & 0.87 \\
\hline \hline
\end{tabular}

Table III. Solution Errors for IBVP 3

\begin{tabular}{ccccc}
\hline \hline \multicolumn{5}{c}{ ENO-4-3 } \\
$N_{c}$ & $\|e\|_{1}$ & $r_{c}$ & $\|e\|_{\infty}$ & $r_{c}$ \\
\hline 64 & $2.464 \mathrm{E}-06$ & & $1.460 \mathrm{E}-05$ \\
128 & $1.665 \mathrm{E}-07$ & 3.89 & $9.783 \mathrm{E}-07$ & 3.90 \\
256 & $1.038 \mathrm{E}-08$ & 4.00 & $6.108 \mathrm{E}-08$ & 4.00 \\
512 & $6.383 \mathrm{E}-10$ & 4.02 & $3.759 \mathrm{E}-09$ & 4.02 \\
\hline \hline
\end{tabular}

Table IV. Solution Errors for IBVP 3

ENO-4-3

\begin{tabular}{ccccc}
$N_{c}$ & $\|e\|_{1}$ & $r_{c}$ & $\|e\|_{\infty}$ & $r_{c}$ \\
\hline 65 & $2.924 \mathrm{E}-05$ & & $9.217 \mathrm{E}-05$ & \\
129 & $1.555 \mathrm{E}-05$ & 0.92 & $4.825 \mathrm{E}-05$ & 0.94 \\
257 & $7.821 \mathrm{E}-06$ & 1.00 & $2.440 \mathrm{E}-05$ & 0.99 \\
513 & $3.910 \mathrm{E}-06$ & 1.00 & $1.224 \mathrm{E}-05$ & 1.00 \\
\hline \hline
\end{tabular}

Table V. Solution Errors for IBVP 3 ENO-4-3-SR

\begin{tabular}{ccccc}
$N_{c}$ & $\|e\|_{1}$ & $r_{c}$ & $\|e\|_{\infty}$ & $r_{c}$ \\
\hline 65 & $2.599 \mathrm{E}-06$ & & $1.153 \mathrm{E}-05$ & \\
129 & $1.552 \mathrm{E}-07$ & 4.11 & $8.644 \mathrm{E}-07$ & 3.78 \\
257 & $9.772 \mathrm{E}-09$ & 4.01 & $5.703 \mathrm{E}-08$ & 3.94 \\
513 & $6.132 \mathrm{E}-10$ & 4.01 & $3.628 \mathrm{E}-08$ & 3.99 \\
\hline \hline
\end{tabular}

Table VI. $L_{\infty}$ Pressure Errors for IBVP 2 ENO-4-3-SR

\begin{tabular}{ccccc}
$N_{c}$ & $x \leq 0.45$ & $r_{c}$ & $x \geq 0.55$ & $r_{c}$ \\
\hline 65 & $7.407 \mathrm{E}-05$ & & $1.853 \mathrm{E}-03$ & \\
129 & $5.665 \mathrm{E}-06$ & 3.71 & $1.831 \mathrm{E}-04$ & 3.34 \\
257 & $3.623 \mathrm{E}-07$ & 3.97 & $1.288 \mathrm{E}-05$ & 3.83 \\
513 & $2.667 \mathrm{E}-08$ & 3.76 & $8.266 \mathrm{E}-07$ & 3.96 \\
\hline \hline
\end{tabular}


a)

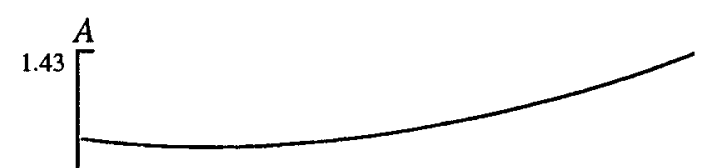

b)

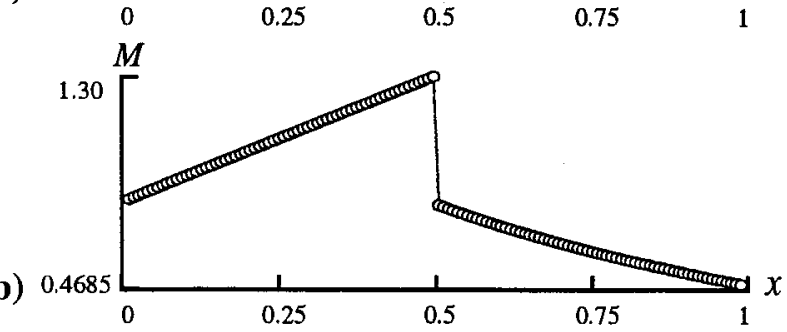

c)

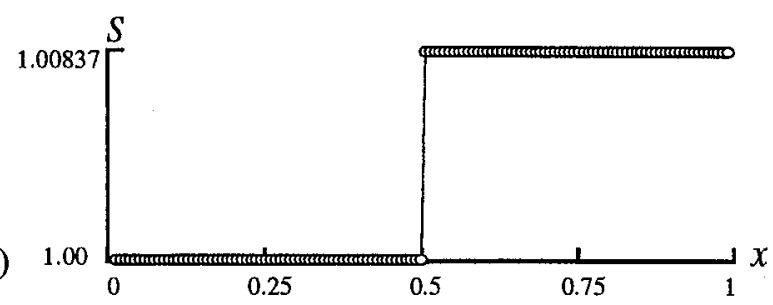

Figure 1: a) Nozzle area. b) Mach number, ENO-4-3. c) $S=P / \rho^{\gamma}$, ENO-4-3.

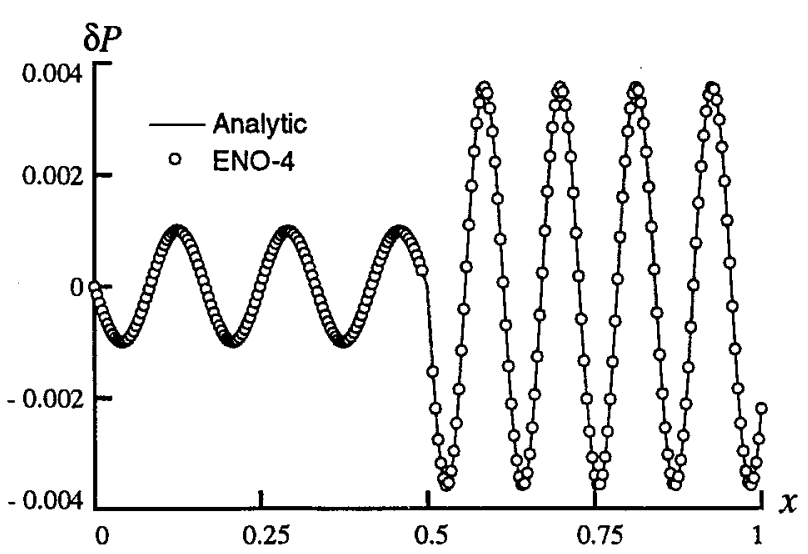

Figure 3: Solution of the sound-shock interaction problem at $t=30 T_{\lambda}$, ENO-4-3.



Figure 4: Pointwise error for the sound-shock interaction problem, ENO-4-3.

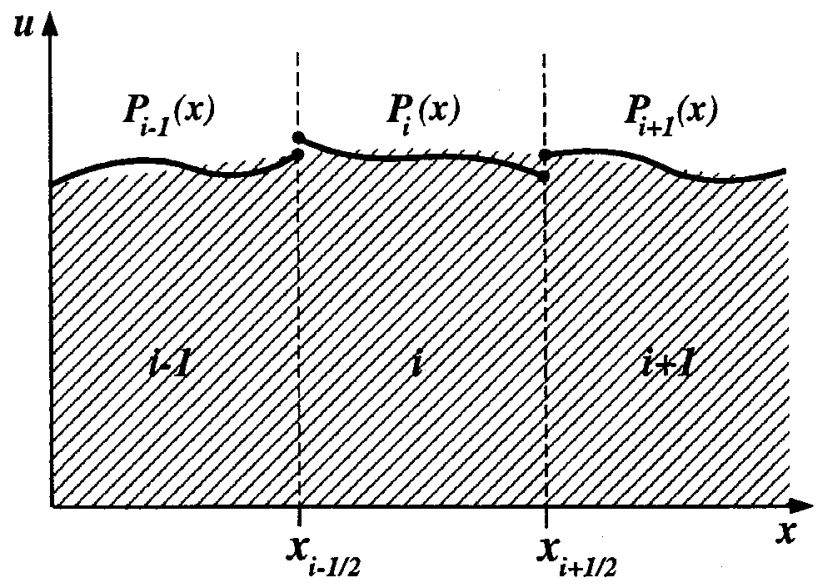

Figure 5: Piecewise polynomial reconstruction. Fluxes are determined by point values at cell faces.

Figure 2: Quasi-1D Nozzle: Entropy error, ENO-4-3. 


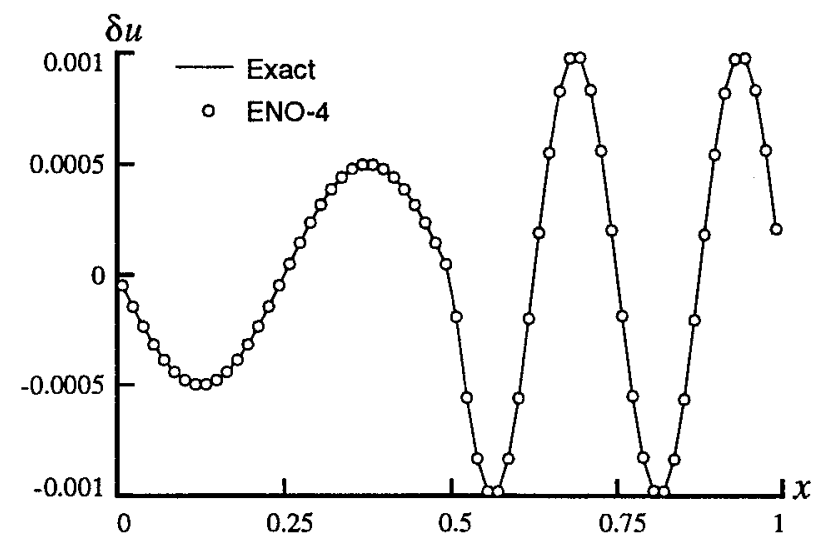

Figure 6: Solution of the IBVP 3 at $t=10 T_{\lambda}$, ENO-4-3.

a)

$\log \mid$ Error $\mid$
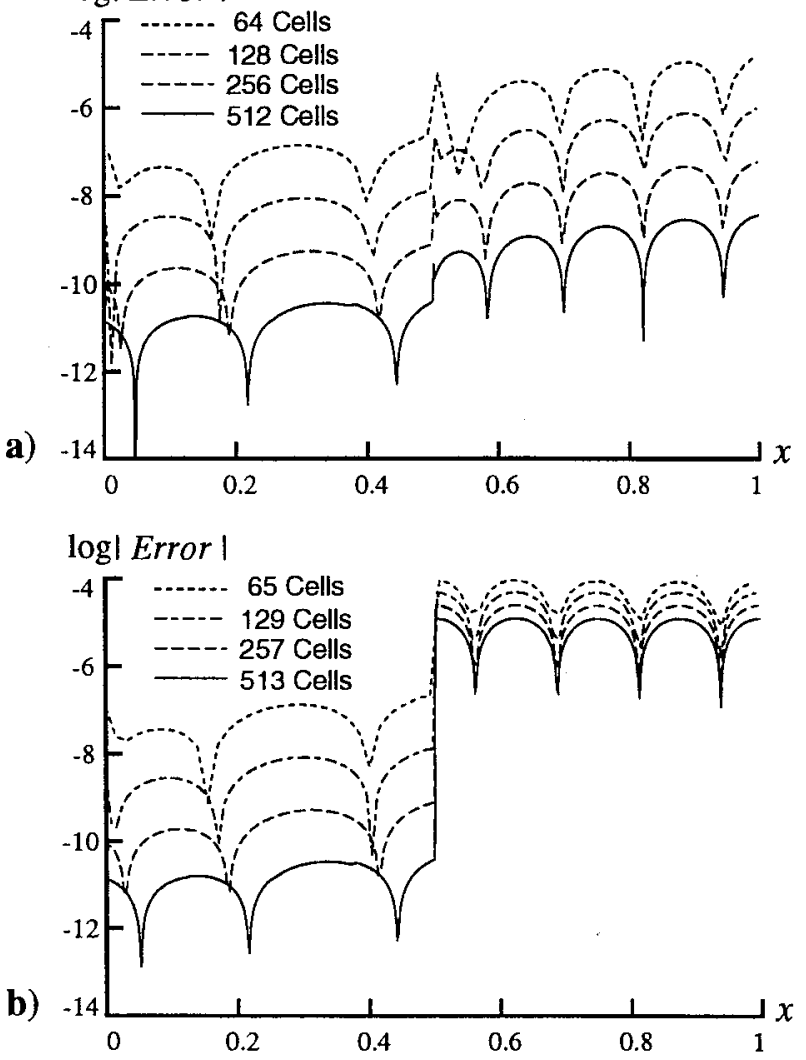

Figure 7: Pointwise error for the IBVP 3, ENO-4-3. a) Discontinuity on a grid point. b) Discontinuity within a cell. a)

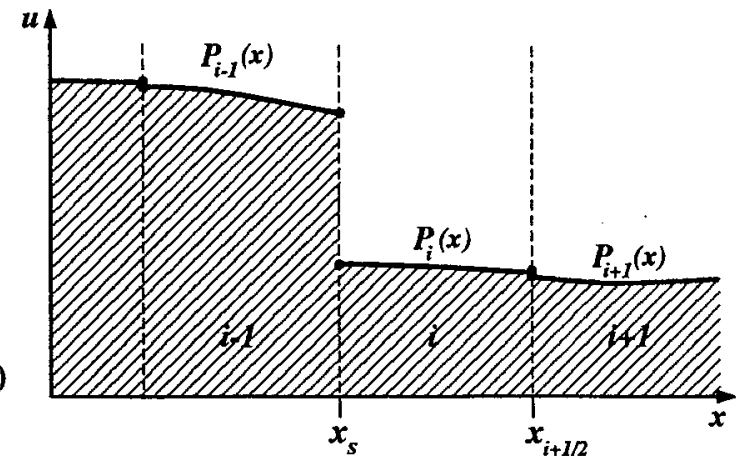

b)

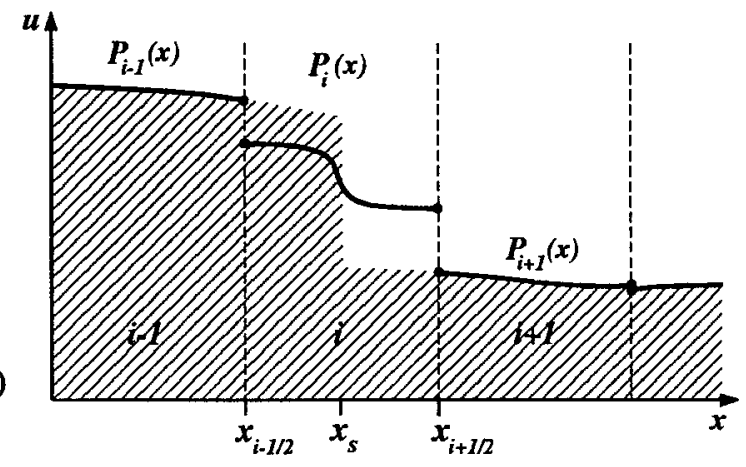

Figure 8: Piecewise polynomial reconstruction for the solution of IBVP 3. a) Discontinuity on a grid point. b) Discontinuity within a cell.

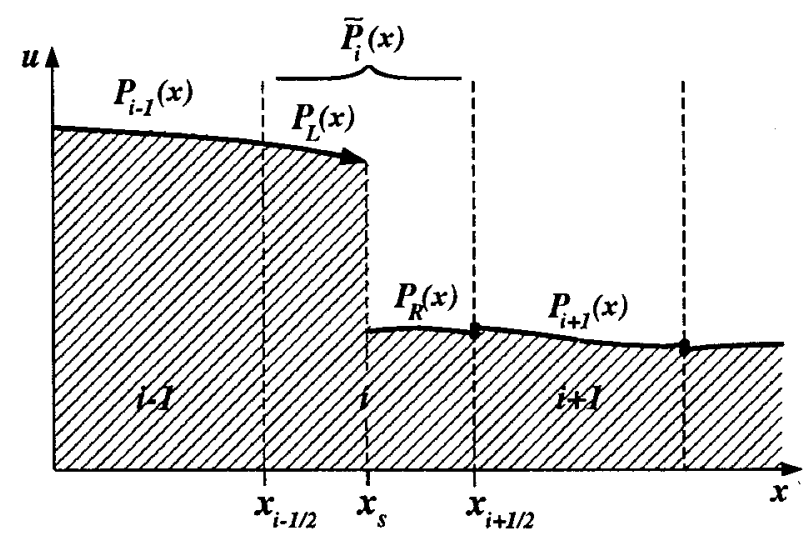

Figure 9: Piecewise polynomial reconstruction for Figure 8b, modified with subcell resolution. 


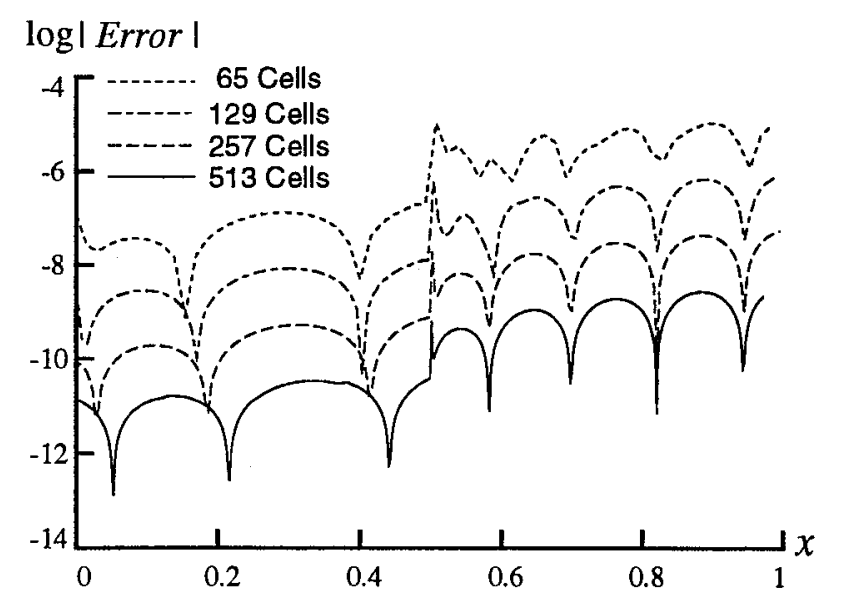

Figure 10: Pointwise error for the IBVP 3 with the discontinuity within a cell, using ENO-4-3-SR.

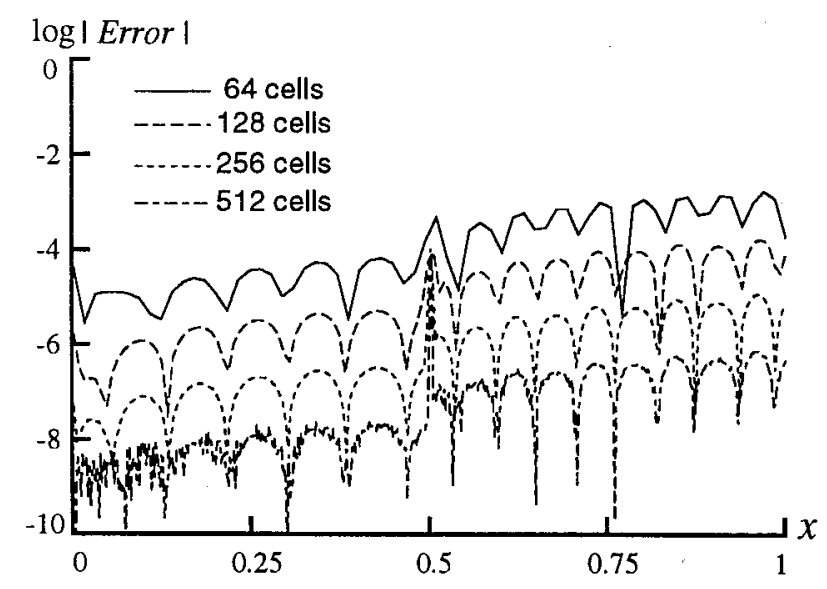

Figure 11: Pointwise error for the sound-shock interaction problem, using ENO-4-3-SR. 



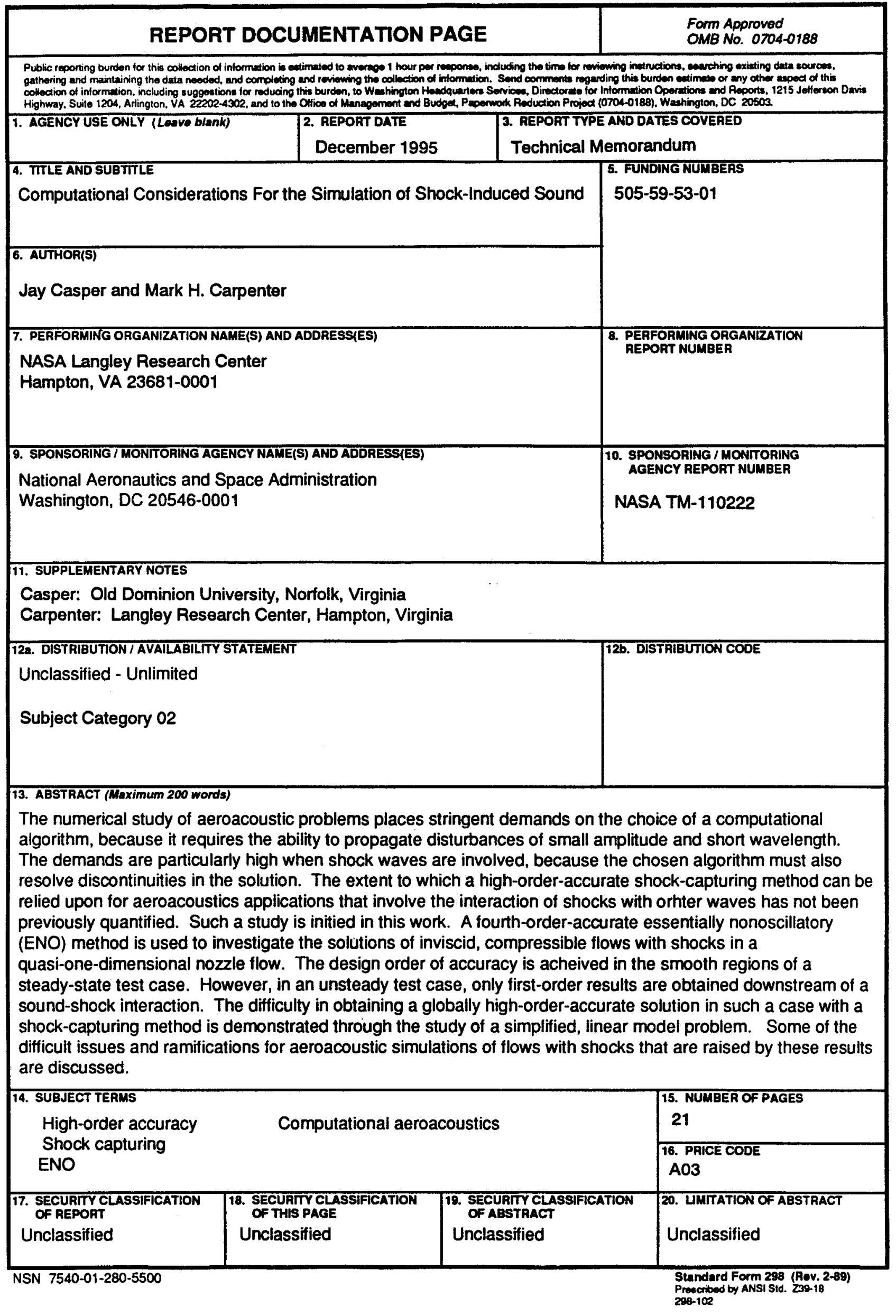



